\title{
Degradación de un efluente textil real asistida por Fe/PVA y materiales residuales magnéticos
}

\section{Real textile effluent degradation assisted by Fe/PVA and magnetic waste}

\author{
Pamela Belém Ramos ${ }^{1}$, Marcela Alejandra Bavio ${ }^{2}$, \\ Gastón Pablo Barreto ${ }^{1}$, Gladys Nora Eyler ${ }^{1}$
}

\author{
${ }^{1}$ INMAT (UNCPBA) y CIFICEN (UNCPBA-CICPBA-CONICET), Av. Del Valle 5737, (B7400JWI), Olavarría, Bue- \\ nos Aires, Argentina. \\ 2 INTELYMEC (UNCPBA) y CIFICEN (UNCPBA-CICPBA-CONICET), Av. Del Valle 5737, (B7400JWI), Olavarría, \\ Buenos Aires,Argentina. \\ e-mail: pamela.ramos@fio.unicen.edu.ar \\ e-mail: mbavio@fio.unicen.edu.ar; gbarreto@fio.unicen.edu.ar; neyler@fio.unicen.edu.ar.
}

\section{RESUMEN}

Los compuestos orgánicos presentes en efluentes industriales son en su mayoría resistentes a los tratamientos biológicos y químicos convencionales. La industria textil ha presentado notables problemas medioambientales vinculados principalmente al uso y la gestión del recurso hídrico, tiene un alto consumo de agua potable y subterránea en sus procesos. Sus aguas residuales son las más contaminantes de los sectores industriales.

Las Procesos Avanzados de Oxidación (PAOs) permiten degradar dichos compuestos de forma no selectiva mediante el ataque de radicales $\bullet \mathrm{OH}$.

En este trabajo se presenta la caracterización y aplicación de diferentes catalizadores para el tratamiento de un efluente textil real (proveniente de una empresa dedicada al teñido y acabado de prendas; ubicada en la provincia de Buenos Aires). Se utilizan tres catalizadores de Fe diferentes: $1^{\circ}$ ) Sintetizado en el laboratorio, $2^{\circ}$ ) Residuo proveniente de la industria metalúrgica y $3^{\circ}$ ) Lana de acero comercial. Los mismos se caracterizan por medio de SEM/EDX. El efluente es caracterizado antes y después del tratamiento, donde el seguimiento de la degradación se mide mediante el \% DQO, pH y contenido de $\mathrm{Fe}^{2+}$ en solución para diferentes condiciones de tratamiento. Se estudia el efecto de catalizadores en el tratamiento aplicado, el cual consiste en una oxidación de la materia orgánica por acción de las especies de Fe en solución y $\mathrm{H}_{2} \mathrm{O}_{2}$, dando un proceso Fenton a pH cercano a la neutralidad.

Los resultados muestran que los tres tipos de catalizadores son eficientes para el proceso de degradación de un efluente textil reales, alcanzando valores de DQO finales aptos para la descarga en una colectora cloacal.

Palabras clave: Catalizadores/potenciadores, efluente real, PAOs, degradación.

\section{ABSTRACT}

Organic compounds present on industrial wastes are mostly resistant to conventional biological and chemical treatments. Textile industry has presented several environmental problems mainly related to the use and management of water resources. It has a high consumption of potable and underground water in its processes. These wastewaters are one of the most polluting of the industrial area.

The Advanced Oxidation Process AOPs allow to decompose organic matter in a non-selectively way by the attack of $\bullet \mathrm{OH}$ radicals.

This work presents the characterization and application of several catalysts for real textile effluent treatment (from an industry dedicated to dyeing and finishing clothes; in Buenos Aires province).Three different iron catalysts are used: $1^{\circ}$ ) Synthesized in a laboratory, $2^{\circ}$ ) Waste from metallurgic industry and $3^{\circ}$ ) commercial steel wool. These are characterized using SEM/EDX. The effluent is characterized before and after treatment, where the degradation is following by measure of $\% \mathrm{COD}, \mathrm{pH}$ and $\mathrm{Fe}^{2+}$ content in solution by condition different of treatment. The effect of catalysts on the applied treatment is studied, which consists of an oxidation 
of organic matter by action of $\mathrm{Fe}$ species and $\mathrm{H}_{2} \mathrm{O}_{2}$ in solution, assuming a Fenton process a $\mathrm{pH}$ neutral.

The results show that the three catalysts are efficient for use in the process of degradation of the real textile effluent. In all cases, the COD final values are suitable for discharge into a sewer system.

Keywords: Catalysts, real effluent, AOPs, degradation.

\section{INTRODUCCIÓN}

La industria textil ha presentado notables problemas medioambientales vinculados principalmente al uso y la gestión del recurso hídrico; tiene un alto consumo de agua potable y subterránea en sus procesos y sus aguas residuales son una de las más contaminantes de todos los sectores industriales [1].

La creciente demanda de la sociedad por la remediación de aguas contaminadas de diversos orígenes, sumada a regulaciones cada vez más estrictas, ha impulsado en los últimos años, el desarrollo de nuevas tecnologías de depuración [2].

La mayoría de los compuestos orgánicos que se encuentran en los efluentes industriales son resistentes a los métodos convencionales de tratamiento químico y biológico [3-6].

Los Procesos Avanzados de Oxidación (PAOs) favorecen la transformación de contaminantes orgánicos con estructuras complejas a sustancias menos tóxicas y más biodegradables [7]. Los $\mathrm{PAOs}\left(\mathrm{O}_{3}, \mathrm{O}_{3} / \mathrm{H}_{2} \mathrm{O}_{2}\right.$, $\left.\mathrm{O}_{3} / \mathrm{UV}, \mathrm{H}_{2} \mathrm{O}_{2} / \mathrm{UV}, \mathrm{O}_{3} / \mathrm{H}_{2} \mathrm{O}_{2} / \mathrm{UV}, \mathrm{Fe}^{2+} / \mathrm{H}_{2} \mathrm{O}_{2}\right)$ se basan en procesos fisicoquímicos capaces de producir cambios fundamentales en la estructura química de los contaminantes a través de la generación in situ de especies radicalarias muy reactivas y de alto poder oxidante $(\bullet \mathrm{OH})$, que pueden obtenerse por procesos catalíticos homogéneos y heterogéneos [8], [9], [10].

La mayoría de los estudios en este campo han sido aplicados para estudiar los mecanismos de degradación mediante PAOs de compuestos específicos, como sustancias colorantes [3], [11-13]; compuestos tóxicos presentes en efluentes industriales [14-16] y algunos productos farmacéuticos [3], [4]. En su mayoría los trabajos realizados corresponden a estudios sobre la degradación de un compuesto específico en efluentes simulados [17] y la determinación de los mecanismos de reacción; sin embargo, son escasos los trabajos de aplicación de PAOs a efluentes reales.

En el proceso conocido como Fenton, se oxida la materia orgánica con peróxido de hidrógeno catalizando con hierro metálico o sales de hierro $\left(\mathrm{H}_{2} \mathrm{O}_{2} / \mathrm{Fe} ; \mathrm{H}_{2} \mathrm{O}_{2} / \mathrm{Fe}^{2+}\right)$ [18], [19]. El proceso Fenton consiste en la adición de sales de $\mathrm{Fe}^{2+}$ en medio ácido para promover la descomposición catalítica del $\mathrm{H}_{2} \mathrm{O}_{2}$ en radicales $\cdot \mathrm{OH}$.

Recientemente se comenzaron a desarrollar estudios sobre reacciones en presencia de catalizadores heterogéneos de $\mathrm{Fe}$ (hierro cerovalente) para la oxidación con $\mathrm{H}_{2} \mathrm{O}_{2}$, lo que se conoce como Fenton heterogéneo. Estos procesos presentan, con respecto al homogéneo, la ventaja de que el catalizador se recupera fácilmente [19-21].

El mecanismo de acción degradativa sobre el efluente está basado en la química de las especies de Fe en solución. Los catalizadores/potenciadores presentan un elevado contenido del $\mathrm{Fe}^{0}$ que proporciona iones $\mathrm{Fe}^{2+}$ generados in situ por reacción con el $\mathrm{H}_{2} \mathrm{O}_{2}$, ecuación (1) [22], [23]. El $\mathrm{Fe}^{2+}$ disuelto se oxida a $\mathrm{Fe}^{3+}$ en presencia de peróxido de hidrógeno formando radicales $\bullet \mathrm{OH}$ ecuación (2).

$$
\begin{aligned}
& \mathrm{Fe}^{0}+\mathrm{H}_{2} \mathrm{O}_{2} \rightarrow \mathrm{Fe}^{2+}+2 \mathrm{HO}^{-} \\
& \mathrm{Fe}^{2+}+\mathrm{H}_{2} \mathrm{O}_{2} \rightarrow \mathrm{Fe}^{3+}+\mathrm{HO}^{\bullet}+\mathrm{HO}^{-}
\end{aligned}
$$

Este aumento de la concentración de especies reactivas actuaría como potenciador del oxidante en la

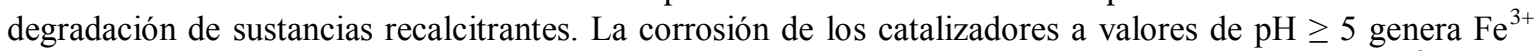
particulado (formación de barros) que enturbian el sistema; sin embargo, parte de estos cationes $\mathrm{Fe}^{3+}$ se en- $^{-}$ cuentran en solución y pueden reducirse rápidamente a cationes $\mathrm{Fe}^{2+}$ por diferentes vías, ecuaciones (3 y 4).

$$
\begin{aligned}
& 2 \mathrm{Fe}^{3+}+\mathrm{Fe}^{0} \rightarrow 3 \mathrm{Fe}^{2+} \\
& \mathrm{Fe}^{3+}+\mathrm{H}_{2} \mathrm{O}_{2} \rightarrow \mathrm{Fe}^{2+}+\mathrm{HO}_{2}+\mathrm{H}^{+}
\end{aligned}
$$


Para estos procesos de oxidación con $\mathrm{H}_{2} \mathrm{O}_{2}$ /catalizadores heterogéneos entre los catalizadores más usados están los siguientes: $\quad \mathrm{Fe}^{\mathrm{o}} ; \quad \mathrm{Fe} / \mathrm{Al}_{2} \mathrm{O}_{3} ; \quad \mathrm{Fe} / \mathrm{SiO}_{2} ; \quad \mathrm{Fe} / \mathrm{MCM} ; \quad \mathrm{Co} / \mathrm{MCM} ; \quad \mathrm{Cu} / \mathrm{MCM}$; $\mathrm{Mx} / \mathrm{n}\left(\mathrm{Al}_{2} \mathrm{O}_{3}\right)_{\mathrm{x}}\left(\mathrm{SiO}_{2}\right)_{\mathrm{y}} \mathrm{mH}_{2} \mathrm{O}$; Fe/ZSM-5; Fe/Carbón Activo; Cu/Carbón Activo [24], [25]

Diversos métodos de síntesis han sido utilizados para preparar partículas de Fe metálico y óxidos de hierro [26][27]. Los métodos químicos son posiblemente los más usados para el desarrollo de nanopartículas metálicas. En general estos métodos se basan en la precipitación de un núcleo constituido por unos pocos átomos metálicos y su posterior crecimiento. Con el fin de bloquear el crecimiento de la partícula estos métodos pueden incluir agentes surfactantes que se adhieren a los átomos metálicos o a la superficie del núcleo, evitando que se unan más átomos, controlando así el crecimiento [28], [29].

El objetivo de este trabajo es estudiar la degradación de un efluente textil real por medio de $\mathrm{H}_{2} \mathrm{O}_{2}$ y catalizadores/potenciadores como fuente de $\mathrm{Fe}^{2+}$. Se realizó la caracterización de los efluentes teniendo en cuenta los parámetros legales permitidos para el volcado cloacal. Se estudió el ataque oxidativo del mismo mediante peróxido y potenciadores/catalizadores que contienen Fe. Para ello se estudian tres tipos de materiales: Fe/PVA sintetizado en el laboratorio, un residuo metalúrgico y lana de acero comercial. Se analiza el efecto de la concentración, tipo de catalizador y de $\mathrm{H}_{2} \mathrm{O}_{2}$ sobre la disminución de DQO.

\section{MATERIALES Y MÉTODOS}

\subsection{Síntesis y caracterización de potenciadores/catalizadores.}

Para sintetizar las partículas de Fe/PVA se adicionaron $0,2 \mathrm{~g}$ de polyvinilalcohol (PVA) a $100 \mathrm{~mL}$ de una solución $0,5 \mathrm{M} \mathrm{FeSO}_{4} \cdot 7 \mathrm{H}_{2} \mathrm{O}$. Previo al agregado del PVA se calentó la solución a $40^{\circ} \mathrm{C}$ con agitación constante. Posteriormente se mantuvo la agitación con un control de temperatura constante de $60^{\circ} \mathrm{C}$ por 20 minutos. Se filtró la mezcla de reacción, y el residuo fue lavado y secado en estufa durante $2 \mathrm{hs} \mathrm{a} 60^{\circ} \mathrm{C}$.

Las muestras se examinaron con un Microscopio Electrónico de Barrido (SEM), equipado con sistema dispersivo de energía (EDS). La observación se realizó bajo el modo de imágenes de electrones secundarios utilizando una tensión de aceleración de 20 kV.

Para la obtención de micrografías se seleccionó una muestra representativa que se adhirió a un porta muestra metálico con recubrimiento de oro (SEM/EDS).

\subsection{Caracterización y degradación del efluente textil.}

El efluente textil real fue obtenido de una industria localizada en el centro de la provincia de Buenos Aires, dedicada al teñido y acabado de prendas Jeans Denim. En este trabajo el efluente fue inicialmente filtrado (banda negra) para remover los sólidos en suspensión (material abrasivo y pelusas). El pH inicial del efluente no fue ajustado y se trabajó a temperatura ambiente $18 \pm 2{ }^{\circ} \mathrm{C}$.

La caracterización del efluente fue realizada mediante métodos normalizados (APHA, 1998): Sólidos Totales (2540 B), Sólidos Volátiles (2540 E), Conductividad (2510 B), Cloruros (4500-Cl B), Sulfatos (4500- $\left.\mathrm{SO}_{4}{ }^{2-} \mathrm{C}\right)$, Fe total (3500-Fe D), dureza (2340 C) y pH.

Se estudió la degradación de la fracción clarificada utilizando diferentes catalizadores (fuente de $\mathrm{Fe}^{2+}$ ) y $\mathrm{H}_{2} \mathrm{O}_{2}$ como oxidante. El proceso de degradación fue realizado en reactores cilíndricos de $50 \mathrm{~mL}$. Para el seguimiento de la degradación se midió la reducción de Demanda Química de Oxígeno (DQO) (APHA, 1998, Método Estándar 5220 D), concentración de $\mathrm{Fe}^{2+}$ (APHA, 1998; Método Estándar 3500 D) y pH para un tiempo de reacción de 300 minutos. En trabajos previos [30] se analizó el rango de valores de las variables más influyentes en el proceso degradativo. La concentración de oxidante usada entre 0 y $0.08 \mathrm{~mol} \mathrm{~L}^{-1}$ y la concentración de catalizador fue 0 y $0,4 \mathrm{~g} \mathrm{~L}^{-1}$. La DQO residual del efluente fue estimada a partir de la ecuación (5):

$\%$ DQO residual $=100-\left(\frac{\text { DQOi-DQOf }}{\text { DQOi }} \times 100\right)$

Donde DQOi es la demanda química de oxígeno inicial del efluente $\left(\mathrm{mgO}_{2} / \mathrm{L}\right)$ y DQOf es la demanda 
química de oxígeno final del efluente $\left(\mathrm{mgO}_{2} / \mathrm{L}\right)$ para 300 min de degradación.

\section{RESULTADOS Y DISCUSIÓN}

\subsection{Caracterización de los diferentes catalizadores.}

La Figura 1 incluye las micrografías SEM y el análisis de composición química correspondiente a los diversos potenciadores utilizados.

partir de las mismas puede determinarse que las partículas de Fe/PVA (Figura 1 a) presentan un tamaño de aproximadamente $1 \mu \mathrm{m}$ y se encuentran uniformemente distribuidas. Las dimensiones de las partículas fueron medidas en imágenes SEM con magnificación 2500x. El residuo metalúrgico (Figura 1 b) presenta láminas de aproximadamente $1 \mathrm{~mm}$ de largo, $20 \mu \mathrm{m}$ de espesor y $200 \mu \mathrm{m}$ de ancho. La lana de acero comercial (Figura 1 c) fue cortada para realizar las mediciones en tramos de $5 \mathrm{~mm}$ de largo. A traves de las micrografías se observan láminas semitubulares de $50 \mu \mathrm{m}$ de diámetro y $10 \mu \mathrm{m}$ de espesor. Las dimensiones de la lana de acero comercial y el residuo metalúrgico fueron determinadas a partir de imágenes SEM con magnificación de 50x.

De acuerdo al análisis químico, el residuo metalúrgico (Figura 1 b) es el que contiene un mayor porcentaje de Fe superficial ( $90 \%$ en peso) comparado con los otros dos potenciadores, además contiene $\mathrm{O}, \mathrm{Si}$, Cr y Mn. El Fe/PVA (Figura 1 a) es el que menor contenido de Fe presenta (35\% en peso) con un alto contenido de $\mathrm{O}$ y $\mathrm{S}$ que son producto del método de síntesis. La lana de acero comercial (Figura $1 \mathrm{c}$ ) contiene $\mathrm{C}$, $\mathrm{Al}$ y $\mathrm{O}$, y presenta un contenido de $\mathrm{Fe}$ cercano al del residuo.

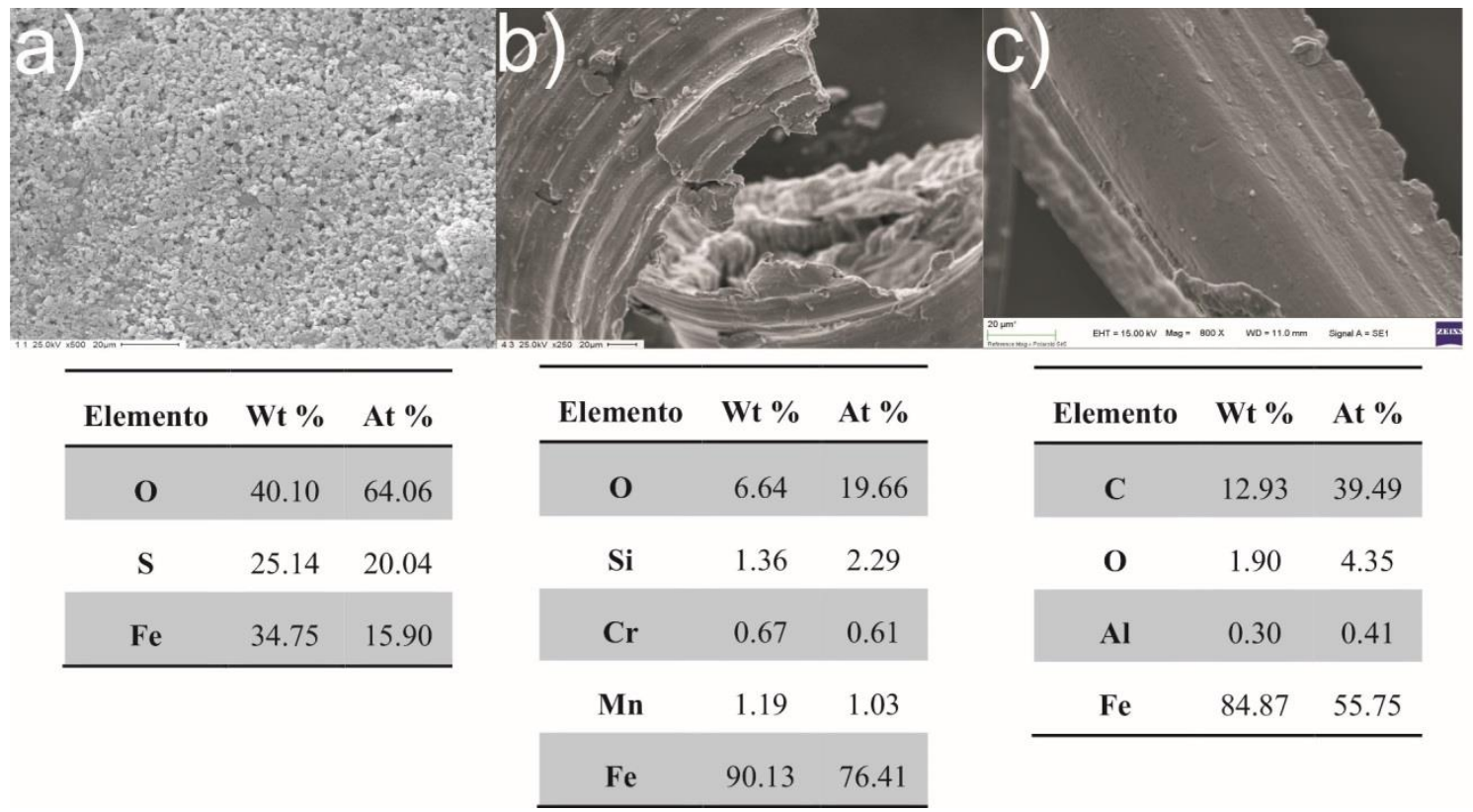

Figura 1: Imágenes SEM y análisis elemental EDS de los potenciadores/catalizadores utilizados. a) Fe/PVA, b) residuo metalúrgico y c) lana de acero comercial.

A efectos de conocer la distribución espacial de Fe, se obtuvieron los mapeos químicos. La Figura 2 muestra los mapeos químicos de $\mathrm{Fe}$ y $\mathrm{O}$ para los tres catalizadores utilizados. Puede observarse que en el catalizador sintetizado (Figura 2 a) los patrones de Fe coinciden con los de $\mathrm{O}$, dando indicios que el Fe puede llegar a encontrarse con un estado de oxidación positivo combinado con el O. Cuando se analizan el residuo (Figura 2 b) y la lana de acero comercial (Figura 2 c) la distribución de $\mathrm{O}$ no coincide totalmente con la de $\mathrm{Fe}$, y, teniendo en cuenta que el contenido de $\mathrm{O}$ es mucho menor que en el Fe/PVA, posiblemente el Fe se encuentre como Fe metálico. 


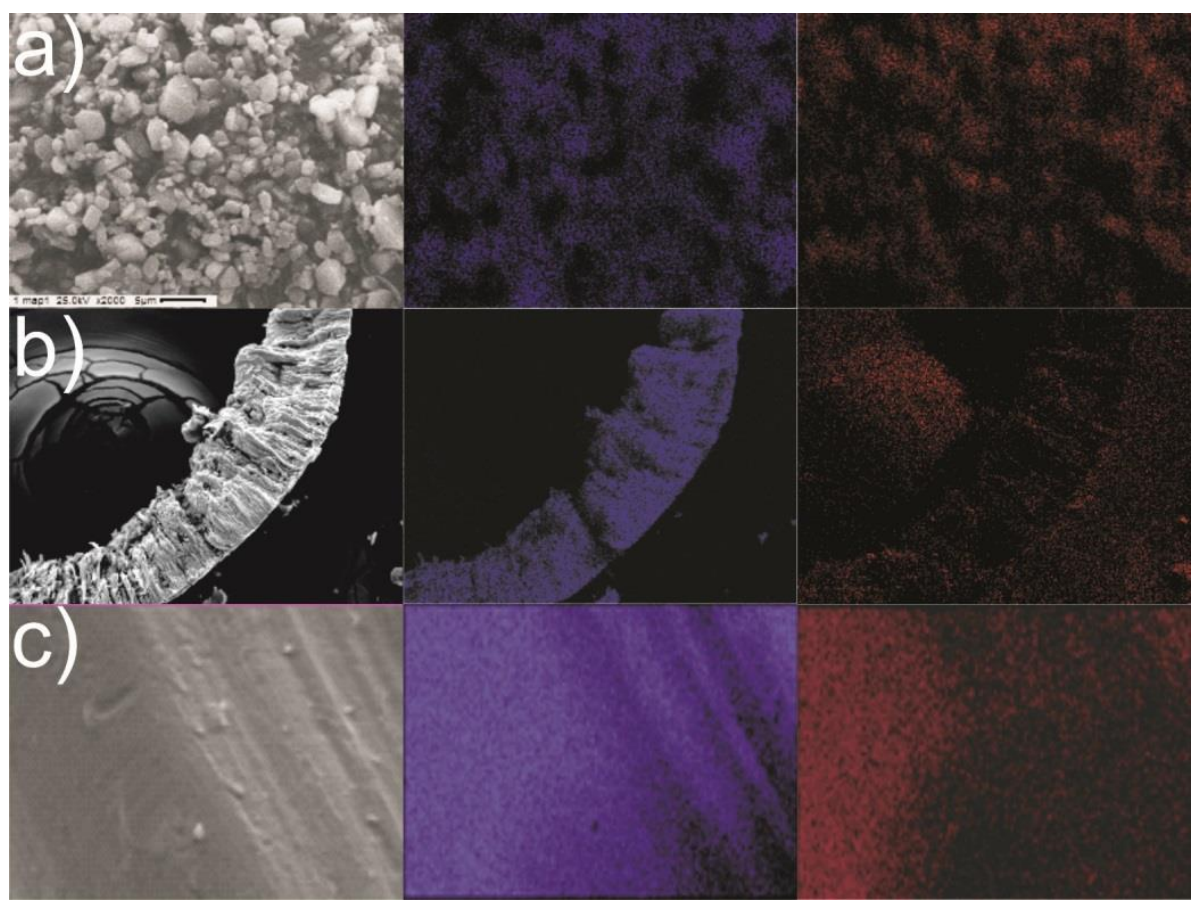

Figura 2: Imágenes SEM y mapeo químico de Fe (violeta) y O (rojo) de los potenciadores/catalizadores utilizados. a) $\mathrm{Fe} / \mathrm{PVA}$, b) residuo metalúrgico y c) lana de acero comercial.

\subsection{Caracterización del efluente textil.}

En la Tabla 1 se muestran las características del efluente textil y se compara con los límites legales establecidos por la Resolución 336/2003 Anexo II (Provincia de Buenos Aires)

Tabla 1: Caracterización del efluente real textil y límites legales.

\begin{tabular}{l|c|c}
\hline \multicolumn{1}{c|}{ Parámetros } & $\begin{array}{c}\text { Efluente } \\
\text { Textil }\end{array}$ & $\begin{array}{c}\text { Límite legal: vertido } \\
\text { en colectora cloacal }\end{array}$ \\
\hline DQO $\left(\mathrm{mg} \mathrm{L}^{-1}\right)^{\mathrm{a}}$ & $\begin{array}{c}\text { Sin filtrar } \\
900-1900 \\
\text { Filtrado } \\
500-1100\end{array}$ & \\
\hline $\mathrm{DBO}_{5} / \mathrm{DQO}^{\mathrm{b}}$ & 0,05 & -- \\
\hline Cloruros $\left(\mathrm{mg} \mathrm{L}^{-1}\right)$ & $0,001-0,009$ & -- \\
\hline Sólidos totales $\left(\mathrm{g} \mathrm{L}^{-1}\right)$ & $2,07-4,24$ & 0,1 \\
\hline Sólidos volátiles $\left(\mathrm{g} \mathrm{L}^{-1}\right)$ & $0,16-2,02$ & -- \\
\hline pH & $6,9-7,4$ & $7-10$ \\
\hline Conductividad $\left(\mathrm{mS} \mathrm{cm}^{-1}\right)$ & $0,20-3,13$ & -- \\
\hline $\mathrm{SO}_{4}{ }^{(}\left(\mathrm{mg} \mathrm{L}^{-1}\right)$ & $20,5-30,6$ & $<1000$ \\
\hline Fe total $\left(\mathrm{mg} \mathrm{L}^{-1}\right)$ & $0,7-0,8$ & -- \\
\hline Dureza $\left(\mathrm{mg} \mathrm{L}^{-1}\right)$ & $160,5-172,4$ & -- \\
\hline Aspecto & Ligera coloración azul & \\
\hline
\end{tabular}

a- Rango de variación en los valores obtenidos experimentalmente de DQO en muestras tomadas durante todo un año. 
b- Relación de biodegradabilidad, un efluente es posible de biodegradar si la relación $\mathrm{DBO}_{5} / \mathrm{DQO}$ da valores mayores a 0,6 [2]. DBO: Demanda Bioquímica de Oxigeno.

Como se observa en la Tabla 1, el efluente tiene una DQO mayor a la que admite la descarga a la red cloacal incluso luego de haber filtrado y eliminado los sólidos. La relación $\mathrm{DBO}_{5} / \mathrm{DQO}$ tiene un valor muy bajo, lo que indica que el efluente no es biodegradable.

\subsection{Degradación del efluente}

La eficiencia en la reducción de DQO fue evaluada de acuerdo a una serie de factores conocidos que afectan al proceso degradativo. En una primera etapa se caracterizó el efluente textil y los catalizadores (fuente de $\mathrm{Fe}^{2+}$ ) y posteriormente se estudió el efecto de cada variable en el proceso de degradación.

\subsubsection{Efecto de la concentración de oxidante $\left(\mathrm{H}_{2} \mathrm{O}_{2}\right)$}

En las siguiente figuras 3, 4, 5 se muestran los resultados obtenidos para una variación de la concentración de $\mathrm{H}_{2} \mathrm{O}_{2}$ entre 0 y $0,08 \mathrm{~mol} \mathrm{~L}^{-1}$ manteniendo fija la cantidad de catalizador agregada en $0,2 \mathrm{~g} \mathrm{~L}^{-1}$ y utilizando el efluente a su $\mathrm{pH}$ inicial y temperatura ambiente. Este contenido de catalizador constante fue determinado mediante los estudios realizados en la variación de DQO residual y la concentración de $\mathrm{Fe}^{0}$ (sección 3.3.2).

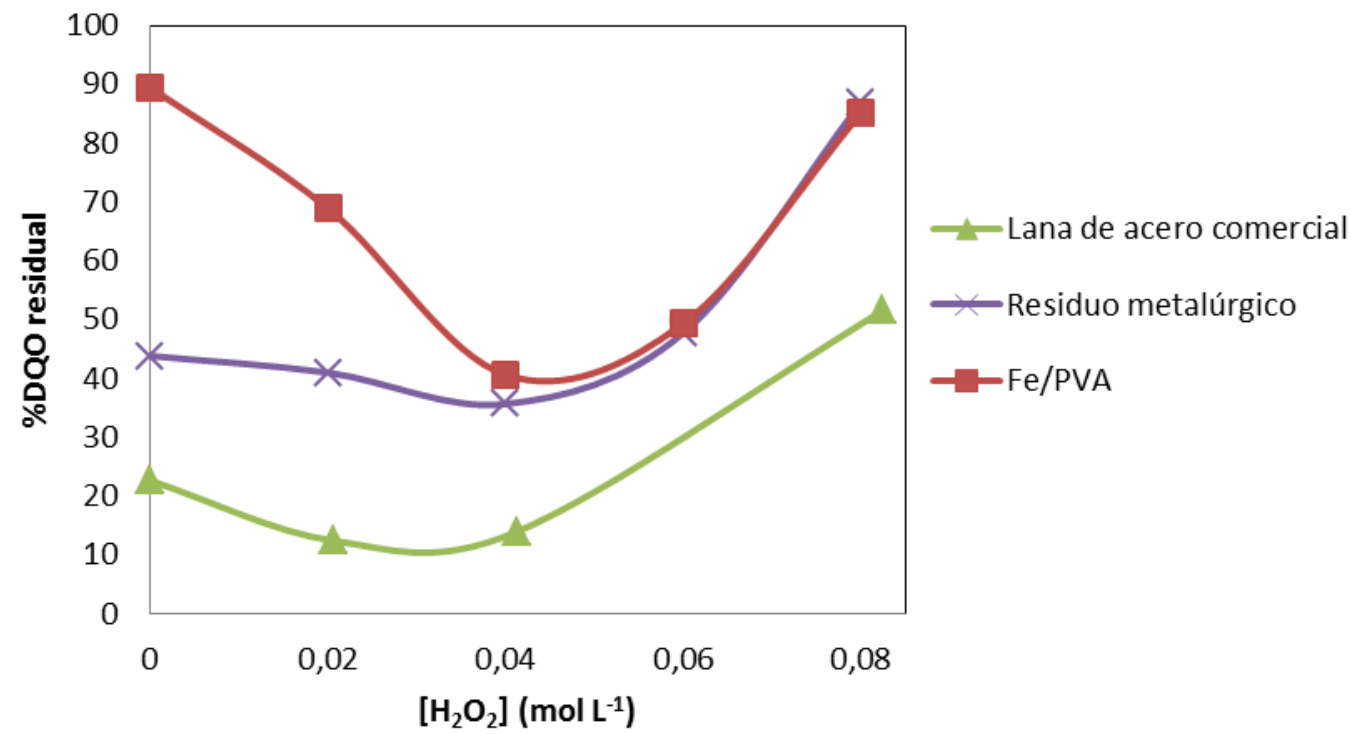

Figura 3: Efecto de la concentración de oxidante sobre \% DQO final del efluente a 300 min para diferentes catalizadores.

En la Figura 3 se observa que se produce una disminucion del \% DQO residual con la concentración óptima de catalizador y sin el agregado de oxidante $\left(\mathrm{H}_{2} \mathrm{O}_{2}\right)$.

Las mayores degradaciones fueron obtenidas para concentraciones de 0,04 molL $^{-1}$ de $\mathrm{H}_{2} \mathrm{O}_{2}$. Inicialmente al incrementar la dosis de $\mathrm{H}_{2} \mathrm{O}_{2}$ aumenta la producción de ${ }^{\circ} \mathrm{OH}$ disponible para atacar las moléculas orgánicas, provocando una disminución en la DQO residual. Sin embargo, al continuar adicionando oxidante, los ${ }^{\circ} \mathrm{OH}$ son consumidos por el $\mathrm{H}_{2} \mathrm{O}_{2}$ para formar un radical menos reactivo, ecuación (6) [31]. Este comportamiento también es observado en procesos Fenton homogéneos [32], [33].

$$
\begin{aligned}
& \mathrm{H}_{2} \mathrm{O}_{2}+\mathrm{HO}^{\bullet} \rightarrow \mathrm{HO}_{2}+\mathrm{H}_{2} \mathrm{O} \\
& \varepsilon_{\mathrm{HO}_{-}}=1,7 \mathrm{~V}<\varepsilon_{\mathrm{HO}^{-}}=2,8 \mathrm{~V}
\end{aligned}
$$

La variación de la concentración de $\mathrm{Fe}^{2+}$ en el efluente degradado 300 minutos para los diferentes catalizadores/potenciadores, se muestra en función de la concentración de oxidante en la Figura 4.

En la figura 4 se observa que durante la degradación de las sustancias recalcitrantes del efluente en presencia 
de oxidante, inicialmente se produce un aumento de la concentracion de $\mathrm{Fe}^{2+}$ en solución, ecuación (1) a concentraciones mayores de $\mathrm{H}_{2} \mathrm{O}_{2}$ el Fe ${ }^{2+}$ es consumido para formar $\mathrm{Fe}^{3+}$, ecuación (2).

Cuando se emplea el catalizador sintetizado Fe/PVA se observa que se necesita más concentración de oxidante para tener disponible el $\mathrm{Fe}^{2+}$ en solución y así lograr las degradaciones observadas en la Figura 3.

A su vez, se observa un máximo de $\mathrm{Fe}^{2+}$ para 0,04 mol L $^{-1}$ de $\mathrm{H}_{2} \mathrm{O}_{2}$ coincidentes con las máximas reducciones de DQO; el efecto observado es similar para los diferentes catalizadores.

$\mathrm{El} \mathrm{pH}$ inicial del efluente antes del tratamiento es de 7,03. En la Figura 5 se observa que en ausencia de oxidante, el pH del efluente aumenta después de los 300 minutos de tratamiento, manteniéndose constante a medida que aumenta la cantidad de $\mathrm{H}_{2} \mathrm{O}_{2}$, este aporte es debido a que el proceso de degradación es mediante la formación de radicales $\mathrm{OH}$ y esto aumenta el $\mathrm{pH}$. El incremento de $\mathrm{pH}$ en el transcurso de la degradación puede explicarse por la formación de $\mathrm{Fe}^{2+}$, ecuación (1). Para concentraciones de hasta 0,04 mol L $\mathrm{L}^{-1} \mathrm{de}_{2} \mathrm{O}_{2}$ y para mayores cantidades de oxidante puede sumarse el consumo del $\mathrm{Fe}^{2+}$ ecuación (2), aportando un efecto mayor al aumento de $\mathrm{pH}$, ya que contribuye a la formación de $\mathrm{OH}^{-}$.

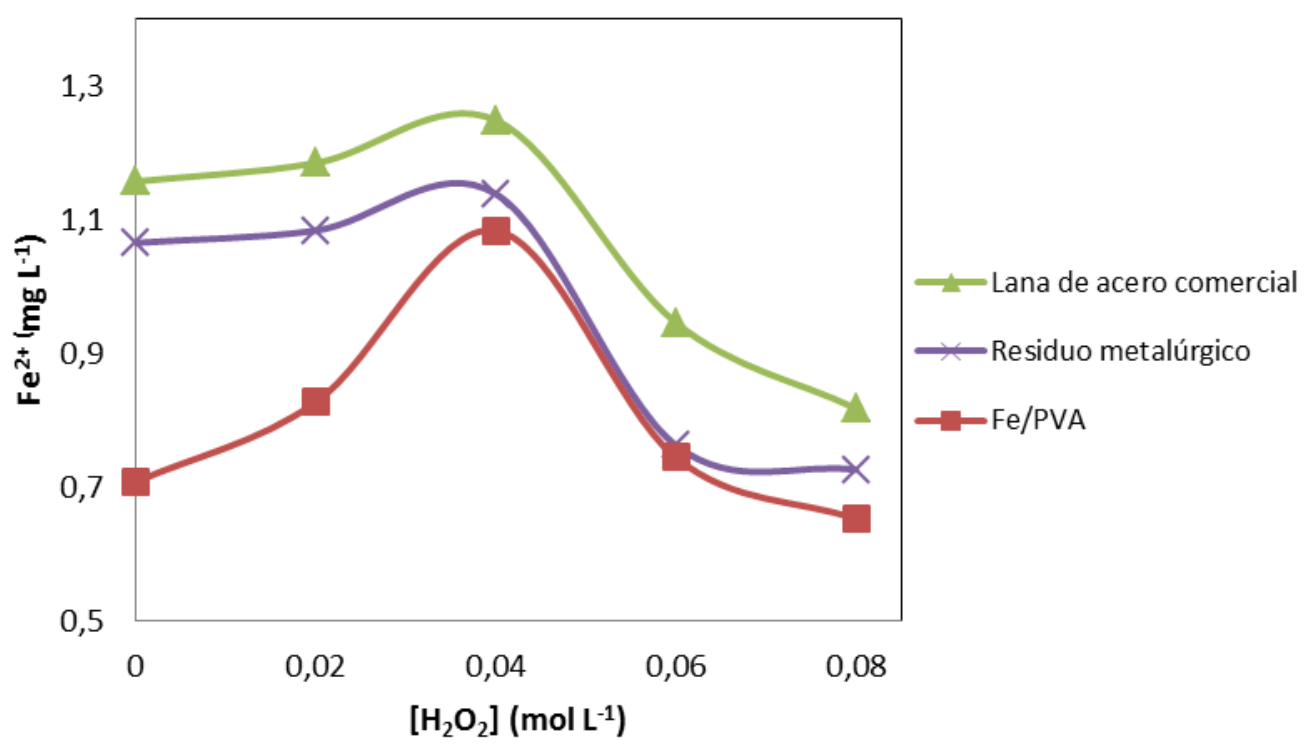

Figura 4: Efecto de la concentración de oxidante sobre $\mathrm{Fe}^{2+}$ en solución para diferentes catalizadores. 


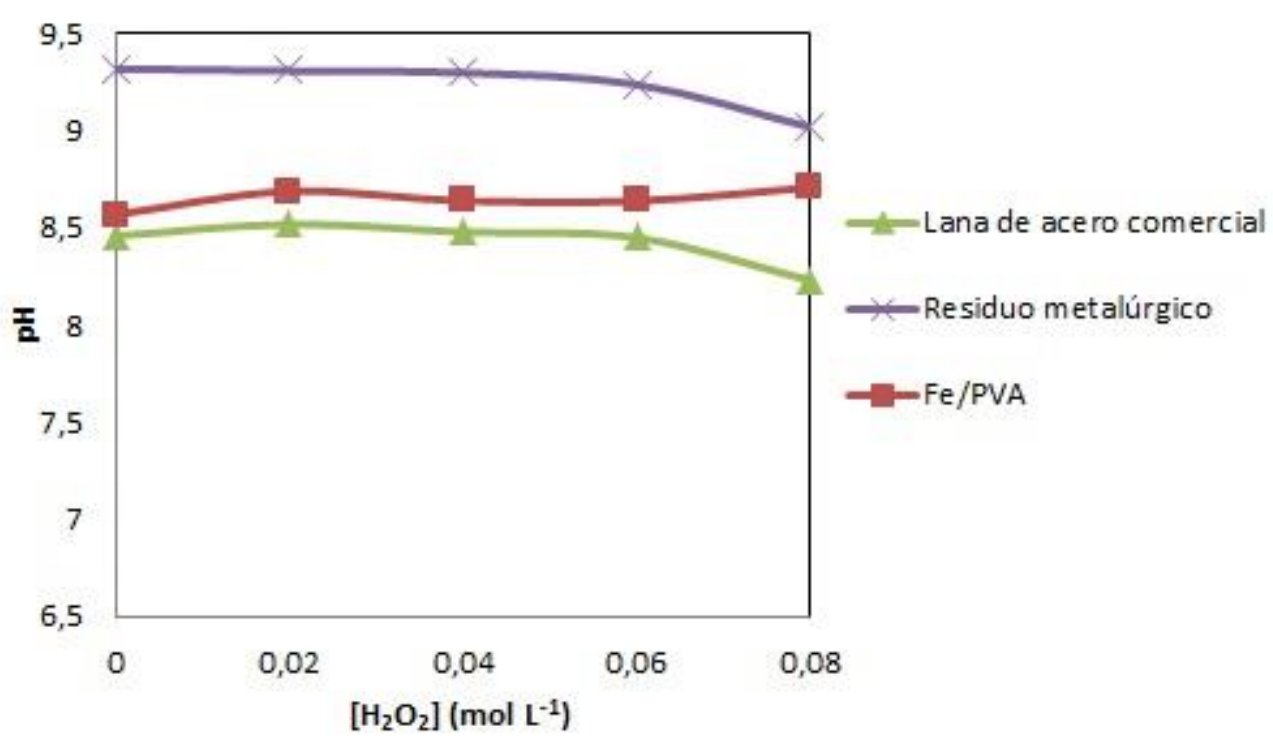

Figura 5: Efecto de la concentración de oxidante sobre el pH final (300 min) para diferentes catalizadores.

El pH inicial del efluente es cercano a la neutralidad (ca. 7,03); el aumento durante el tratamiento oscila entre 1 y 2,5 unidades de $\mathrm{pH}$. Si bien es conocido que el aumento de $\mathrm{pH}$ a valores superiores a 5 promueve la precipitación de $\mathrm{Fe}^{3+}$ formando barros [34], en la empresa la producción de efluente por día es de 250 $\mathrm{m}^{3}$, por lo que sería inviable aplicar procesos de acidificación y neutralización para su descarga.

\subsubsection{Efecto de la concentración de catalizadores/potenciadores.}

A continuación se muestran los resultados obtenidos para la degradación del efluente con una variación de la concentración de catalizadores agregada entre 0 y $0,4 \mathrm{~g} \mathrm{~L}^{-1}$, manteniendo fija la concentración de $\mathrm{H}_{2} \mathrm{O}_{2}$ en $0,04 \mathrm{~mol} \mathrm{~L}^{-1}$ y utilizando el efluente a su pH inicial y a temperatura ambiente. El valor de concentración de $\mathrm{H}_{2} \mathrm{O}_{2}$ de $0,04 \mathrm{~mol} \mathrm{~L}^{-1}$ se mantuvo fijo en esta experiencia debido a que es la concentración óptima obtenida en la sección anterior, donde se encontraron los mínimos valores de \% DQO residual (Figura 3) y los máximos valores de cantidad de $\mathrm{Fe}^{2+}$ (Figura 4).

En la Figura 6, se puede observar que la lana de acero comercial es el catalizador/potenciador que reduce la DQO del efluente en mayor medida para los 300 minutos de tratamiento. 


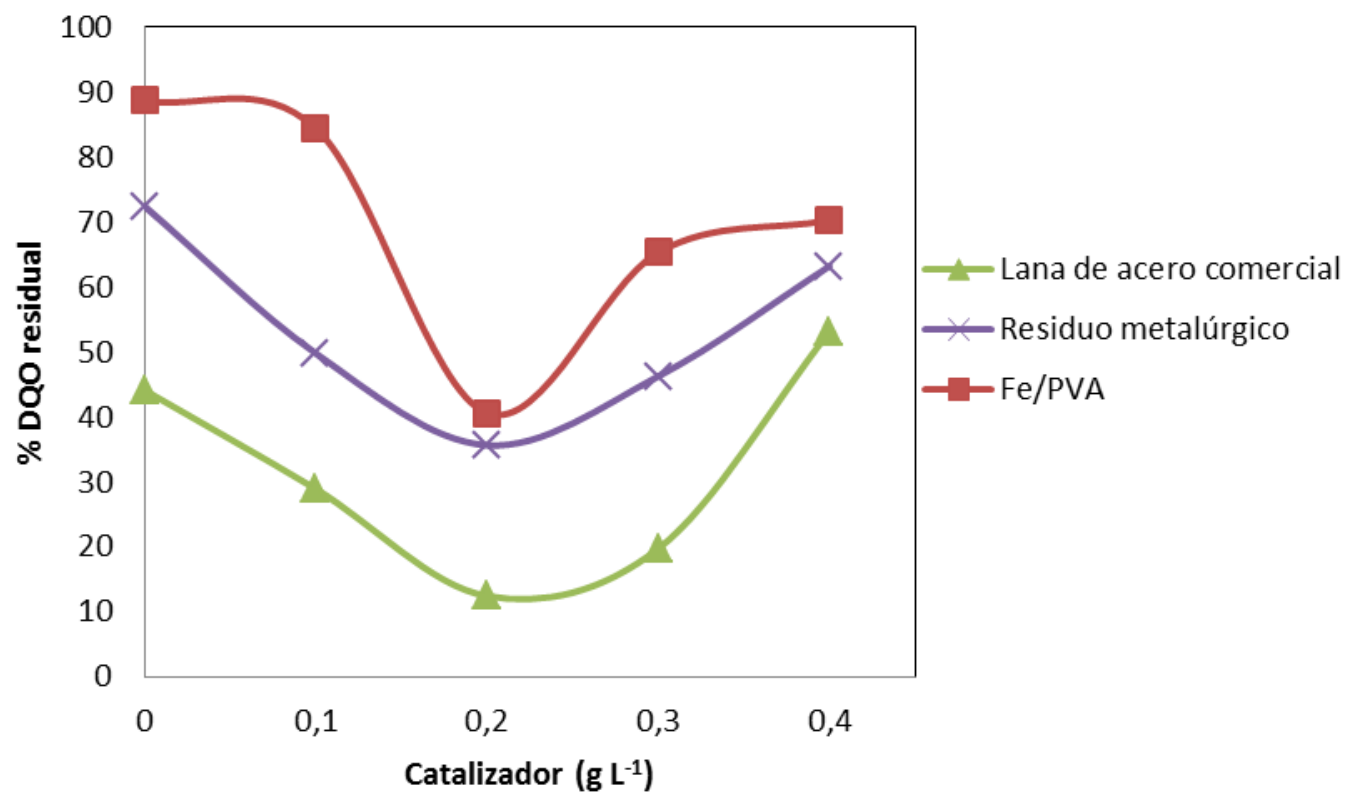

Figura 6: Efecto de la concentración de catalizador sobre el \%DQO residual (300 min) para diferentes catalizadores.

Las mayores degradaciones (Figura 6) fueron obtenidas para $0,2 \mathrm{~g} \mathrm{~L}^{-1} \mathrm{de}$ los diferentes catalizadores, efecto similar al observado en la variación de $\mathrm{H}_{2} \mathrm{O}_{2}$. Cuando la concentración de catalizadores agregada es inferior a $0,2 \mathrm{~g} \mathrm{~L}^{-1}$, el \% DQO residual aumenta, esto puede deberse a que el $\mathrm{Fe}^{2+}$ en solución sería consumido para la generación de ${ }^{\circ} \mathrm{OH}$ (ecuación 2). Para cantidades mayores a $0,2 \mathrm{~g} \mathrm{~L}^{-1}$ se evidencia que la cantidad de $\mathrm{Fe}^{2+}$ en solución disminuye muy poco (Figura 6) lo cual podría deberse a que a altas concentraciones de $\mathrm{Fe}^{2+}$ se puede producir a su vez el atrapamiento de radicales ${ }^{\circ} \mathrm{OH}$ (ecuación 7) generando un aumento en el \% DQO residual. Resultados similares fueron encontrados por Mei-Feng at al. 2011, utilizando $\mathrm{Fe}^{0}$ como catalizador para la degradación de Rodamina, obteniendo un valor óptimo de $\mathrm{Fe} / \mathrm{H}_{2} \mathrm{O}_{2}$ y luego una disminución en la tasa de degradación.

$$
\mathrm{HO}^{\bullet}+\mathrm{Fe}^{2+} \rightarrow \mathrm{Fe}^{3+}+\mathrm{HO}^{-}
$$

$\mathrm{El} \mathrm{pH}$ aumenta a lo largo del tratamiento favorecido por las ecuaciones 2, 3 y 6 (Figura 8). Este efecto es mayor a menores concentraciones de catalizador. $\mathrm{El} \mathrm{pH}$ inicial del efluente es cercano a la neutralidad ( $c a$. 7,03 ), el aumento durante el tratamiento oscila entre 1,3 y 2 unidades de $\mathrm{pH}$. 


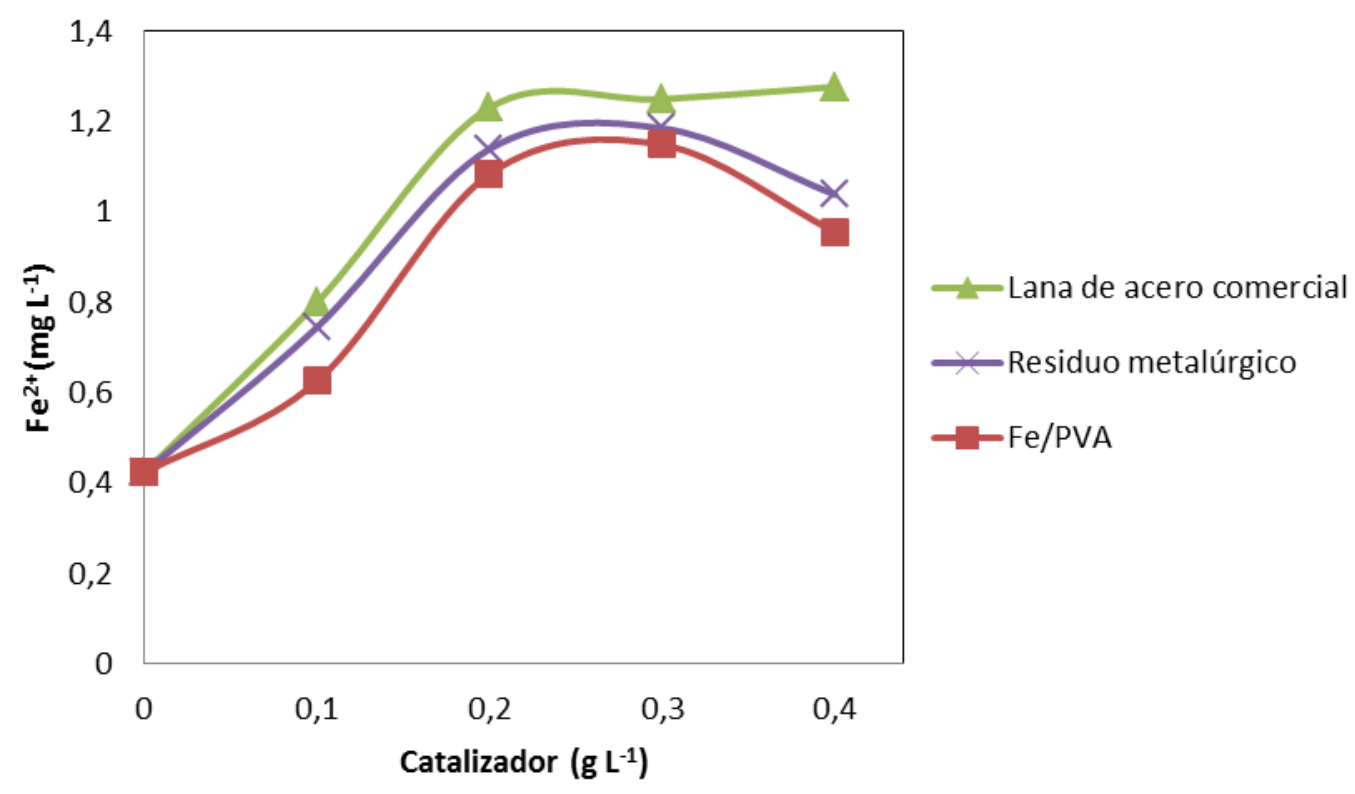

Figura 7: Efecto de la concentración de catalizador sobre la concentración de $\mathrm{Fe}^{2+}$ para diferentes catalizadores.

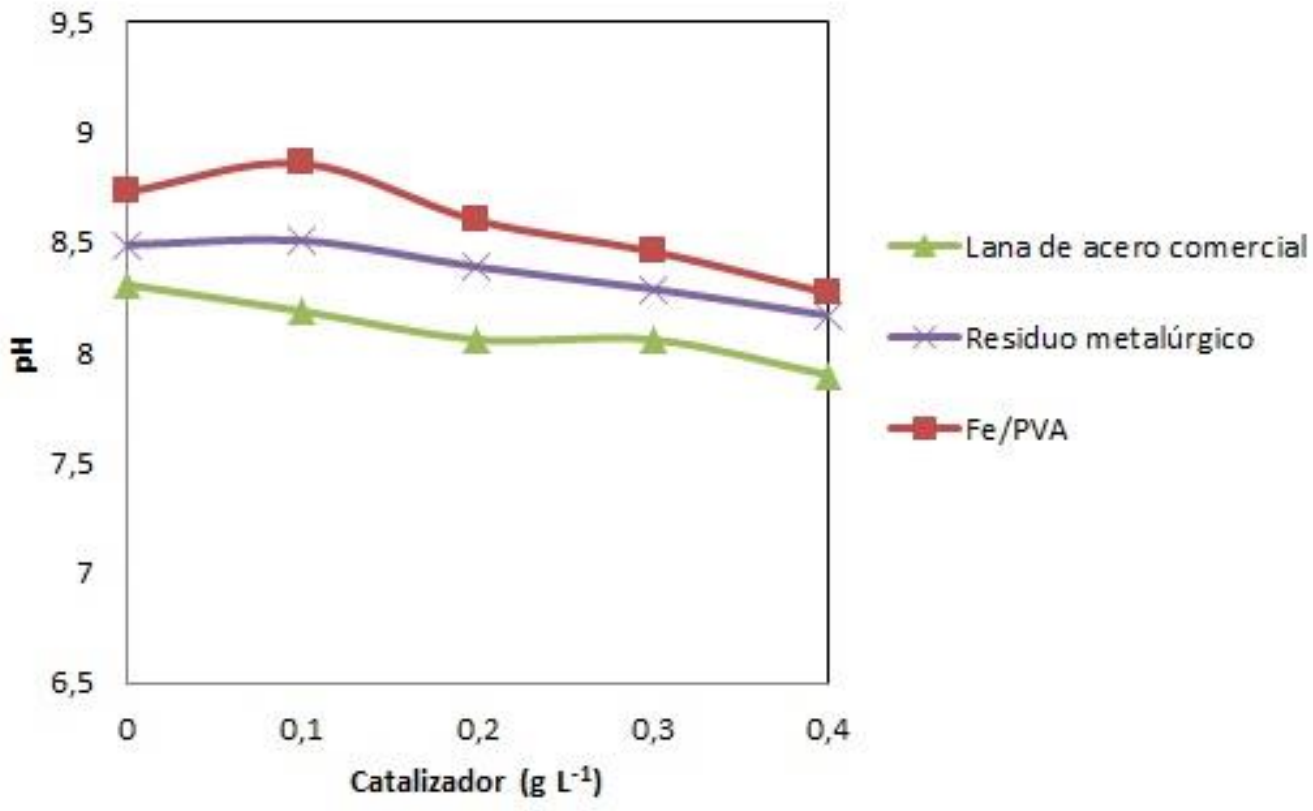

Figura 8: Efecto de la concentración diferentes catalizadores sobre el pH del efluente a 300 min.

\section{CONCLUSIONES}

Se analizó el efecto de la concentración de $\mathrm{H}_{2} \mathrm{O}_{2}$ para los tres potenciadores/catalizadores, a un tiempo de 300 minutos de proceso de degradación. Los mejores resultados fueron obtenidos con una concentración de peróxido de $0,04 \mathrm{~mol} \mathrm{~L}^{-1}$ para la lana de acero comercial, quedando un \% DQO residual de $14 \%\left(150 \mathrm{mgO}_{2}\right.$ $\mathrm{L}^{-1}$ ), dando de esta manera valores óptimos para su descarga en desagües cloacales. El estudio realizado como función de la concentración de $\mathrm{Fe}^{2+}$, da como mejores catalizadores a la lana de acero comercial y el residuo metalúrgico, con una concentración de $\mathrm{Fe}^{2+}$ de 1,25 y $1,14 \mathrm{mg} \mathrm{L}^{-1}$, respectivamente. Cuando se analizan los contenidos totales de Fe según los reportes EDS, estos valores se correlacionan con los 
porcentajes encontrados, sin embargo la concentración de $\mathrm{Fe}^{2+}$ para el PVA es similar (13\% menor) que en la lana de acero, dando indicios que el contenido de Fe en el material no es tan significativo como lo es la disponibilidad del mismo.

Se estudió el efecto de la concentración de catalizador sobre la degradación del efluente, encontrándose un valor óptimo para los tres materiales analizados de $0,2 \mathrm{~g} \mathrm{~L}^{-1}$.

A su vez, la variación de $\mathrm{pH}$ es menor a 2,5 unidades utilizando cualquiera de los tres catalizadores, este aumento es consecuencia de las reacciones que se producen en el proceso de degradación.

El uso de este tipo de proceso para la degradación de un efluente real, proporciona valores apropiados de descarga que cumplen con la legislación vigente de la Provincia de Buenos Aires. En función de las posibilidades y los análisis económicos de la empresa se puede optar por un tipo u otro de catalizador, dependiendo de los costos de producción y de la posibilidad de reutilizar residuos de otras industrias, o bien usar materiales que son comerciales y económicos.

\section{AGRADECIMIENTOS}

Este trabajo ha sido financiado por la Comisión de Investigaciones Científicas de la Provincia de Buenos Aires (CICPBA), el CONICET, la Facultad de Ingeniería y la Secretaria de Ciencia, Arte y Tecnología (SECAT) de la UNCPBA. G.P. Barreto y M. A. Bavio son investigadores de CONICET.G. N. Eyler es investigador asociado a CICPBA. P. B. Ramos es becaria de CONICET.

\section{BIBLIOGRAFÍA}

[1] MICHAEL-KORDATOU,I., MICHAEL,C., DUAN, X., et al., "Dissolved effluent organic matter: Characteristics and potential implications in wastewater treatment and reuse applications," Water Research, vol. 77, pp. 213-248, 2015.

[2] BLANCO JURADO, J. "Degradación de un efluente textil real mediante procesos Fenton y Foto-Fenton", Universidad Politecnica de Catalunya, 2009.

[3] OLLER, I., MALATO, S., SÁNCHEZ PÉREZ, J. A. "Combination of Advanced Oxidation Processes and biological treatments for wastewater decontamination--a review.," The Science of the total environment, v. 409, n. 20, pp. 4141-66, Sep. 2011.

[4] NOONPUI, S., THIRAVETYAN,P., NAKBANPOTE, W., et al., "Color removal from water-based ink wastewater by bagasse fly ash, sawdust fly ash and activated carbon," Chemical Engineering Journal, vol. 162, no. 2, pp. 503-508, Aug. 2010.

[5] SPONZA, D. T., OZTEKIN, R. "Removals of some hydrophobic poly aromatic hydrocarbons (PAHs) and Daphnia magna acute toxicity in a petrochemical industry wastewater with ultrasound in Izmir-Turkey," Separation and Purification Technology, v. 77, n. 3, pp. 301-311, Mar. 2011.

[6] TARAN, M. "Utilization of petrochemical wastewater for the production of poly(3-hydroxybutyrate) by Haloarcula sp. IRU1”, Journal of hazardous materials, v. 188, n. 1-3, pp. 26-8, Apr. 2011.

[7] GOGATE, P. R., PANDIT, A. B. "A review of imperative technologies for wastewater treatment I : oxidation technologies at ambient conditions", v. 8, n. 3, pp. 501-551, 2004.

[8] HEREDIA, C. L., SHAM, E. L. "Tartrazine degradation by supported $\mathrm{TiO}_{2}$ on magnetic particles", Matéria (R.J), v.23, n.3, pp. 668-675, 2015.

[9] DOMÈNECH, X., JARDIM, W. F., LITTER, M. I. “Capitulo 1: procesos avanzados de oxidación para la eliminación de contaminantes", In: Programa Iberoamericano de ciencia y tecnología para el desarrollo (CYTED), CYTED., M. A. Blesa, Ed. La Plata, pp. 3-25, 2001.

[10] AZBAR, N., YONAR, T., KESTIOGLU, K. "Comparison of various advanced oxidation processes and chemical treatment methods for COD and color removal from a polyester and acetate fiber dyeing effluent", Chemosphere, v. 55, n. 1, pp. 35-43, Apr. 2004.

[11] BERGAMINI, R. B. M., AZEVEDO, E. B., ARAÚJO, L. R. R. "Heterogeneous photocatalytic degradation of reactive dyes in aqueous $\mathrm{TiO}_{2}$ suspensions: Decolorization kinetics," Chemical Engineering Journal, v. 149, n. 1-3, pp. 215-220, Jul. 2009.

[12] GARCÍA MONTAÑO, J. "Combination of advanced oxidation processes and biological treatments for commercial reactive azo dyes removal”, Universidad Autonoma de Barcelona, 2007.

[13] SHU, H. "Degradation of dyehouse effluent containing C . I . Direct Blue 199 by processes of 
ozonation, $\mathrm{UV} / \mathrm{H}_{2} \mathrm{O}_{2}$ and in sequence of ozonation with $\mathrm{UV} / \mathrm{H}_{2} \mathrm{O}_{2}$ ", v. 133, pp. 92-98, 2006.

[14] WU,S., WALLACE, S., BRIX, H., et al., "Treatment of industrial effluents in constructed wetlands: challenges, operational strategies and overall performance.," Environmental pollution (Barking, Essex: 1987), v. 201, pp. 107-20, Jun. 2015.

[15] CÁCERES VAZQUEZ, J. Evaluación analítica y optimización de procesos de oxidación avanzada en planta piloto solar, Tesis de D.Sc, Universidad de Almería, España, 2002.

[16] ZHANG,S., ZHAO, X., NIU, H., et al., "Superparamagnetic $\mathrm{Fe}_{3} \mathrm{O}_{4}$ nanoparticles as catalysts for the catalytic oxidation of phenolic and aniline compounds", Journal of hazardous materials, v. 167, n. 1-3, pp. 560-6, Aug. 2009.

[17] LUCAS, M. S. "Decolorization of the azo dye Reactive Black 5 by Fenton and photo-Fenton oxidation", Dyes and Pigments ,v. 71, pp. 236-244, 2006.

[18] BABUPONNUSAMI, A., MUTHUKUMAR, K. "A review on Fenton and improvements to the Fenton process for wastewater treatment”, Journal of Environmental Chemical Engineering, v. 2, n. 1, pp. 557-572, Mar. 2014.

[19] BREMNER, D. H., BURGESS, A. E., HOULlEMARE, D., "Phenol degradation using hydroxyl radicals generated from zero-valent iron and hydrogen peroxide", Applied Catalysis B: Environmental, v. 63, n. 1-2, pp. 15-19, Mar. 2006.

[20] XU, L., WANG, J. "A heterogeneous Fenton-like system with nanoparticulate zero-valent iron for removal of 4-chloro-3-methyl phenol", Journal of hazardous materials, v. 186, n. 1, pp. 256-64, Feb. 2011.

[21] HOU, M. F., LIAO, L., ZHANG, W. D., et al, "Degradation of rhodamine B by Fe(0)-based Fenton process with $\mathrm{H}_{2} \mathrm{O}_{2}$ ", Chemosphere, v. 83, n. 9, pp. 1279-83, May 2011.

[22] GRČIĆ, I., ŠIPIĆ, A., KOPRIVANAC, N., et al., "Global parameter of ultrasound exploitation (GPUE) in the reactors for wastewater treatment by sono-Fenton oxidation", Ultrasonics Sonochemistry, v. 19, n. 2, pp. 270-279, 2012.

[23] ORTIZ DE LA PLATA, G. B., ALFANO, O. M., CASSANO, A. E. "2-Chlorophenol degradation via photo Fenton reaction employing zero valent iron nanoparticles", Journal of Photochemistry and Photobiology A: Chemistry, v.233, pp. 53-59, 2012.

[24] JIMÉNEZ, J. J. R. Hacia un uso sostenible de los recursos naturales. Universidad Internacional de Andalucía, 2008.

[25] WANG, N., ZHU, L., WANG, M., et al., "Sono-enhanced degradation of dye pollutants with the use of $\mathrm{H}_{2} \mathrm{O}_{2}$ activated by $\mathrm{Fe}_{3} \mathrm{O}_{4}$ magnetic nanoparticles as peroxidase mimetic", Ultrasonics sonochemistry, v. 17, $\mathrm{n}$. 1, pp. 78-83, Jan. 2010.

[26] FERNANDEZ, K. C. Síntesis y Caracterización de Nanopartículas Magnéticas, Tesis de D.Sc, UNAM, México, 2013.

[27] GARCÍA, C. L., ROJAS, S. S. "Síntesis de nanopartículas metálicas con un método de química verde", In: 11 Encuentro International e Interdisciplinario en nanociencia y Nanotecnologia, Universidad Juárez Autónoma de Tabasco, México, n. 3, pp. 2-3.

[28] MAKSIMOVIĆ, V. M., PAVLOVIĆ, L. J., JOVIĆ, B. M., et al., "Electrodeposition of Fe powder from acid electrolytes," Journal of the Serbian Chemical Society, v. 73, n. 8-9, pp. 861-870, 2008.

[29] SANTANA, N. C. "Síntesis Y Caracterización De Nanopartículas De Metal Hexacianoferrato Obtenidas en Microemulsiones Inversas”, Revista Iberoamericana de Polímeros, v. 11, n. 7, pp. 457-470, 2010.

[30] RAMOS, P. B., VITALE, P., EYLER, G. N. Evaluación de las cineticas de degradación de un efluente textil real, Clicap, Mendoza, 2015.

[31] FENG, F., XU, Z., LI, X., et al., "Advanced treatment of dyeing wastewater towards reuse by the combined Fenton oxidation and membrane bioreactor process", Journal of Environmental Sciences, v. 22, n. 11, pp. 1657-1665, Nov. 2010.

[32] ERTUGAY, N., ACAR, F. N. "Removal of COD and color from Direct Blue 71 azo dye wastewater by Fenton's oxidation: Kinetic study," Arabian Journal of Chemistry, v. 10, pp. S1158-S1163, 2017.

[33] PÉREZ, M., TORRADES, F., DOMENECH, X., et al., "Fenton and photo-Fenton oxidation of textile effluents", Water Research, v. 36, n. 11, pp. 2703-2710, 2002. 
[34] WANG, N., ZHENG, T., ZHANG,G., et al., "A review on Fenton-like processes for organic wastewater treatment", Journal of Environmental Chemical Engineering, v. 4, n. 1, pp. 762-787, 2016. 